\title{
Prescription Changes During Geriatric Care Episodes: A Trend Analysis Conducted in Sweden
}

\author{
Marianne Reimers $^{1}$ (1) $\cdot$ Maria Eriksdotter $^{1} \cdot$ Åke Seiger $^{1} \cdot$ Johan Fastbom $^{1}$
}

Published online: 19 February 2018

(c) The Author(s) 2018. This article is an open access publication

\begin{abstract}
Background The number of drugs prescribed for old people has been rising in recent decades. With increasing age and multiple medications, the risk of complications and drug prescription complexity increases. Multiple changes to prescriptions could be a factor that improves treatment quality.

Objectives Our objective was to investigate trends in drug prescriptions and factors that contribute to prescription changes. Specific objectives were to find out whether high numbers of prescription changes are significantly correlated with age, sex, comorbidity, length of care episode, and number of drugs.

Methods Data were extracted from geriatric clinic records in Stockholm in 2005, 2010, and 2015. Indicators for good drug therapy were used to assess the effects of prescription changes on quality, using an index of inappropriate drug use (IDU). Data were analyzed with Student's $t$-test, PR test, Wilcoxon's rank sum test, and linear regression.

Results Patients had more comorbidities and used more drugs but had shorter hospital stays and significantly fewer prescription changes in 2015 than in 2005. Length of care episode was significantly associated with prevalence of prescription changes. Our model showed that, for each day by which the length of care episode decreased, the number of prescription changes decreased by $8 \%$. The number of prescription changes was negatively correlated to the IDU index score.

Conclusions The study showed that more prescription changes were associated with longer care episodes and
\end{abstract}

\footnotetext{
Marianne Reimers

marianne.reimers.wessberg@ki.se

1 Karolinska Institutet, Stockholm, Sweden
}

improved drug prescribing quality as per the IDU index. Given prescription changes are regarded as a quality factor in geriatric care, quality may have decreased along with the length of care episodes between 2005 and 2015.

\section{Key Points}

Study patients had significantly shorter hospital stays and fewer prescription changes in 2015 than in 2005.

More prescription changes were significantly associated with improved drug-prescribing quality and longer care episodes.

Care quality may have decreased along with the length of care episodes during the 2005-2015 study period.

\section{Introduction}

In recent decades, debate regarding polypharmacy among old people has been intense [1]. Haider et al. [2] showed that the prevalence of polypharmacy (use of five or more drugs) in individuals aged $\geq 77$ years increased from 18 to 42\% during the 1992-2002 period. Guthrie et al. [3] reported that, in Scotland between 1995 and 2010, the percentage of adults with five or more prescriptions nearly doubled, from 11.4 to $20.8 \%$, and the percentage with ten or more prescriptions tripled, from 1.7 to $5.8 \%$. Wastesson et al. [4] showed that polypharmacy among people aged $\geq 75$ years in Sweden increased from $43.1 \%$ in 2006 to $46.6 \%$ in 2013 and that prevalence increased with age. In 
2013 in Sweden, $20 \%$ of patients aged $\geq 65$ years received five or more drugs, and $55 \%$ of patients aged $\geq 95$ years received five or more drugs.

The risk of complications rises with increasing age and more medications. Aging, per se, brings physiological and functional changes [5] that lead to increased sensitivity to drugs [6]. Receiving more drugs increases the risk of inappropriate drug use (IDU) such as the potential for drug-drug interactions [7, 8]. A lower quality of drug use among the elderly can lead to drug-related illness [9-12].

Both the extent and the pattern of drug use differs between females and males: According to SNAC-K (Swedish National Study on Aging and Care in Kungsholmen), in Stockholm in 2007 [1], 51.4\% of the females and $33.2 \%$ of the males received analgesics. The corresponding percentages for psychotropic drugs were $48.3 \%$ for females and $30.8 \%$ for males. Females had a significantly higher prevalence of drug prescriptions than did men and would therefore be at higher risk for complications and in greater need of interventions.

Wickop et al. [13] compared three protocols to detect potentially inappropriate medication (PIM) in a German hospital. They found the prevalence of PIM varied between protocols and that all three showed a higher prevalence of PIM during the hospital stay than before and after the care episode.

Studies on the impact of geriatric care on quality in drug prescription are scarce and report conflicting results. A Danish study of effects of a geriatric care episode [14] showed that anticholinergic and benzodiazepine prescriptions were reduced significantly (and to a greater degree) in the geriatric ward than in other medical wards. In contrast, Dauphinot et al. [15] reported that the use of anticholinergic and sedative drugs increased during stays in three French geriatric clinics. Larsen et al. [16] reported that geriatric care resulted in relatively few changes in medication prescriptions.

Consequently, the overall aim of this study was to investigate trends in long-term drug prescriptions and factors that contribute to prescription changes. Specific objectives were to (1) find out whether a high number of prescription changes were significantly correlated with age, sex, comorbidity, length of care episode, and number of drugs and (2) determine whether the findings were representative for geriatric patients in general.

\section{Methods}

\subsection{Subjects and Setting}

Study subjects were patients with pneumonia who were admitted to a geriatric clinic (Brommageriatriken,
Stockholm, Sweden) with 129 inpatient beds during 2005, 2010, and 2015 .

To be included, patients had to have had a diagnosis code of J189.9 (pneumonia) according to the International Statistical Classification of Diseases and related Health Problems, tenth revision, Clinical Modification (ICD-10$\mathrm{CM})$ registered as the primary diagnosis.

For patients treated for pneumonia more than once, only the first care episode was included because we did not want to miss changes that were likely to have already been made during a previous geriatric care episode. Patients who died during the care episode were excluded because drug prescriptions at discharge was an important factor in the analyses. We do not believe this led to a selection of less serious cases, since patients with both one and several care episodes were included.

\subsection{Data Collection}

In 2005, patient age and sex, the length of their care episode, and their comorbidities [17] were extracted from Cosmic (Cambio Healthcare Systems, Stockholm, Sweden), which was the data system in use at the clinic at that time. In 2010 and 2015, data were extracted from TakeCare (CompuGroup Medical, Stockholm, Sweden), which was the data system in use at the clinic as of 2008. Similar clinical data were registered in both systems. The different information systems did not influence data collection or results concerning regularly used drugs.

All regularly used drugs at admission and discharge and prescription changes during the care episode that were still current at discharge were also extracted.

Drugs related to the primary diagnosis of pneumonia were not included in the analysis as they were used only temporarily. Drugs administered as needed were likewise not included because they were not consistently registered throughout the study periods.

Sweden's National Board of Health and Welfare $[18,19]$ proposed "indicators for good drug therapy among the elderly," and these indicators were used to assess quality effects due to prescription changes: (1) presence of long-acting benzodiazepines, (2) presence of anticholinergic drugs, (3) presence of duplicate drugs, (4) concurrent use of three or more psychotropic drugs, (5) prevalence of drug combinations that may cause class $\mathrm{C}$ drug-drug interactions [those that may lead to altered effects or adverse drug reactions (ADRs) but may be managed with individual dosage and/or plasma concentration monitoring of the drug], and (6) prevalence of drug combinations that may cause class D drug-drug interactions (those that may lead to serious clinical consequences in the form of severe ADR or lack of effect or is otherwise difficult to manage with individual dosage). 
Based on these indicators, the present study developed an index of IDU to measure the quality of drug prescribing. The index score was the sum of the instances of at least one occurrence of each of the six aforementioned indicators. Therefore, a lower index score during a care episode indicated an improvement in prescribing quality.

\subsection{Data Analyses}

Data were analyzed using Stata, version 10 (College Station, TX, USA). A $p$ value $<0.05$ was considered statistically significant.

We used Student's $t$ test to evaluate differences between years regarding age, length of care episode, and number of drugs at admission and discharge; the PR test to calculate the percentage of females; and Wilcoxon's rank sum test to evaluate the Charlson's comorbidity index (CCI) score and the IDU index score. We used linear regression analyses to evaluate the correlation between endpoint prescription changes and age, sex, length of care episode, comorbidity, number of drugs, and the IDU index score.

All factors were entered into the study's statistical model and considered to be potential confounders or effect modifiers.

To evaluate the representativeness of patients with pneumonia for all geriatric patients in the clinic, we compared the age, sex, and length of care episode of those in the study with those of all patients in the clinic each year.

\section{Results}

Over the 3 years studied, 146, 134, and 140 patients, respectively, were included. The proportion of females was 55,53 , and $57 \%$, respectively (Table 1 ).

Between 2005 and 2015, the length of the care episode decreased consistently and comorbidities assessed with the CCI increased significantly. The CCI score was higher for males than for females each year: 2.3 for males versus 1.6 for females in 2005; 2.9 versus 1.7 in 2010; and 3.0 versus 1.8 in 2015 .

The number of drugs at admission and discharge was higher in 2010 and 2015 than in 2005, whereas the number of drug changes increased between 2005 and 2010 and decreased thereafter. The changes in IDU index score were significant when comparing 2015 with both 2010 and 2005 (Table 2).

Regression analyses were performed to estimate the correlation between the factors studied and the endpoint prescription changes. The analyses showed that the only factor correlated with drug changes during all 3 years was the length of the care episode. In 2015, comorbidity was the factor most significantly correlated to drug changes.
However, the length of the care episodes contributed (Table 3). Age and sex were not significantly associated.

We further examined whether any of the variables could be confounders or effect modifiers of the correlation between drug changes and length of care episodes. Adjusting for age cancelled the association in 2010, but this effect disappeared when we studied data from all 3 years.

When analyzing data from all 3 years, we found a crude correlation between drug changes and length of care episodes, with a coefficient of 0.0816 . When adjusting for the other factors, the correlation was virtually unchanged (0.0807). Thus, according to our model, for each day by which the length of care episode shortened, the prevalence of drug changes decreased by $8 \%$.

To evaluate the representativeness of the study, we compared the demographics of patients in the study with those of all patients admitted to the entire geriatric clinic in 2010 and 2015 ( $n=3591$ and $n=4436$, respectively). Results showed that patients in our study were slightly older: the mean age in 2010 was 85.2 years in the study and 83.3 years in the clinic, whereas the mean age in 2015 was 84.0 years in the study and 83.9 years in the clinic. The average length of the care episode was similar in the study and entire clinic populations: in 2010, patients were treated for 11.19 days in the study versus 10.8 days in the clinic, and in 2015, patients were treated for 9.9 days in the study versus 9.6 days in the clinic. The percentage of females was lower in the study than in the entire clinic population: in 2010, the percentage of females was $53.0 \%$ in the study versus $64.3 \%$ in the clinic, whereas in 2015 , the corresponding numbers were 57.1 versus $62.5 \%$.

To better understand the difference in percentage of females between the study and the entire clinic, we measured the percentage of chronic obstructive pulmonary disease (COPD), which constitutes a risk for pneumonia. Our analysis revealed that COPD in the entire clinic was more common among males than females (10.3 males vs. 9.4\% females in 2010 and 11.0 males vs. 9.3\% females in 2015), although differences were moderate. In the study, COPD was more common in both sexes $(25.7 \%$ males vs. $20 \%$ females in 2010 and $28.3 \%$ males vs. $22.5 \%$ females in 2015). In addition, overrepresentation of males in 2010 was $9.6 \%$ in the clinic and $28.5 \%$ in the study. In 2015 , the overrepresentation of males was $18.3 \%$ in the clinic and $25.8 \%$ in the study.

\section{Discussion}

Our study shows that patients at the geriatric clinic had significantly more comorbidities and received more drugs but had significantly shorter hospital stays and fewer 
Table 1 Characteristics of the study subjects in 2005, 2010, and 2015

\begin{tabular}{llll}
\hline Characteristics & 2005 & 2010 & 2015 \\
\hline$N$ & 146 & 134 & 140 \\
Age (years) & $84.3 \pm 7.0$ & $85.2 \pm 6.8$ & $84.0 \pm 8.7$ \\
Sex, \% females $(n)$ & $54.8(80)$ & $53.0(71)$ & $57.1(80)$ \\
Length of care episode (days) & $12.2 \pm 8.0$ & $10.8 \pm 5.9$ & $9.9 \pm 4.8$ \\
Mean CCI score & 1.9 & 2.2 & 2.4 \\
Number of drugs at admission & $6.4 \pm 3.8$ & $8.3 \pm 3.9$ & $8.8 \pm 4.3$ \\
Number of drugs at discharge & $6.3 \pm 3.8$ & $7.9 \pm 3.9$ & $8.8 \pm 4.3$ \\
Number of drug changes & $1.88 \pm 1.80$ & $2.13 \pm 1.86$ & $1.1 \pm 1.4$ \\
Mean IDU index at admission & 0.50 & 0.57 & 0.66 \\
Mean IDU index at discharge & 0.49 & 0.41 & 0.70 \\
Mean IDU index, change during care episode & -0.01 & -0.16 & 0.04 \\
\hline
\end{tabular}

Data are presented as mean \pm standard deviation unless otherwise indicated

CCI Charlson's comorbidity index, IDU inappropriate drug use

Table 2 Significance of mean differences between the years concerning the factors studied

\begin{tabular}{lccc}
\hline Tests of differences between years & 2010 vs. 2005 & 2015 vs. 2010 & 2015 vs. 2005 \\
\hline Age, mean difference in years $(p$ value, $t$ test $)$ & $0.94(0.260)$ & $-1.20(0.206)$ & $-0.27(0.777)$ \\
Proportion of females, difference in $\%,(p$ value, PR test $)$ & $0.018(0.762)$ & $-0.042(0.489)$ & $-0.023(0.689)$ \\
Length of care episode, mean difference in days $(p$ value, $t$ test $)$ & $-1.44(0.848)$ & $-0.92(0.160)$ & $-2.35(0.003)^{*}$ \\
Comorbidity $(p$ value, Wilcoxon's rank sum-test, $z)$ & $1.51(0.130)$ & $2.40(0.016)^{*}$ & $4.09(<0.001)^{*}$ \\
Number of drugs at admission, mean difference $(p$ value, $t$ test $)$ & $1.87(<0.001)^{*}$ & $0.56(0.259)$ & $2.43(<0.001)^{*}$ \\
Number of drugs at discharge, mean difference $(p$ value, $t$ test $)$ & $1.63(0.001)^{*}$ & $0.85(0.086)$ & $2.48(<0.001)^{*}$ \\
Drug changes, mean difference $(p$ value, $t$ test) & $0.26(0.2140)$ & $-1.03(<0.001)^{*}$ & $-0.78(<0.001)^{*}$ \\
IDU index, mean difference $(p$ value, Wilcoxon's rank sum-test, $z)$ & $-2.79(0.005)^{*}$ & $4.29(<0.001)^{*}$ & $1.70(0.088)$ \\
\hline
\end{tabular}

Data are presented as mean \pm standard deviation unless otherwise indicated

$I D U$ inappropriate drug use

$* p<0.05$

prescription changes in 2015 than 10 years earlier. We also found that a higher number of prescription changes correlated with longer hospital stays and a lower IDU index score. Moreover, the association between the geriatric care episode and the quality of drug prescription was positive during 2010 but negative during 2015 as per the IDU index score.

The pattern over time between 2005, 2010, and 2015 showed significant changes in several factors. The number of drugs at admission and the number of comorbidities increased, whereas the length of the care episode and the prevalence of drug changes decreased. Thus, patients had more comorbidities and received more drugs but were treated for a shorter period of time and geriatricians made fewer changes to their prescriptions in 2015 than in 2005.

When comparing the patients in our study with those from the entire geriatric clinic, we identified an overrepresentation of males in our study. More males than females had COPD at admission, which is one explanation for the overrepresentation. Our data also showed that the males in our study had more comorbidities, as indicated by CCI score, possibly increasing the need for hospital care for their pneumonia, which may also have contributed to the sex distribution.

In contrast to our expectations, we found that only one factor-length of care episode-was significantly (positively) correlated to prescription changes over the studied years. Although comorbidity also correlated to drug changes in 2015, prescription changes decreased along with the length of the care episode between 2010 and 2015, whereas, at the same time, comorbidity increased. When all 3 years were studied together, the only significant factor was again the length of the care episode. We find it surprising that comorbidity did not systematically correlate to the prescription changes and that age and number of drugs did not correlate at all.

There may be various reasons for this consistent correlation. Either (1) patients are subject to fewer prescription 
Table 3 Regression analyses on the correlation between length of care episode, comorbidities, inappropriate drug use index, and the endpoint prescription changes

\begin{tabular}{lcccc}
\hline Correlation with endpoint drug changes & Coefficient & SD & $p$ value & 95\% CI \\
\hline 2005 & & & & \\
$\quad$ Length of care episode & 0.071 & 0.018 & $<0.001^{*}$ & $0.036-0.106$ \\
$\quad$ Comorbidity & -0.046 & 0.055 & 0.396 & -0.061 to 0.154 \\
IDU index change & -0.659 & 0.463 & 0.157 & -1.574 to 1.612 \\
2010 & 0.106 & 0.026 & $<0.001^{*}$ & $0.055-0.158$ \\
Length of care episode & 0.012 & 0.062 & 0.844 & -0.112 to 0.136 \\
Comorbidity & 0.699 & 0.326 & $0.034^{*}$ & $0.055-1.344$ \\
IDU index change & & & & \\
2015 & 0.053 & 0.025 & $0.033^{*}$ & $0.004-0.102$ \\
$\quad$ Length of care episode & 0.182 & 0.056 & $0.001^{*}$ & $0.072-0.293$ \\
Comorbidity & 0.077 & 0.512 & 0.880 & -0.936 to 1.091 \\
IDU index change & & & & \\
All data & 0.082 & 0.013 & $<0.001^{*}$ & $0.057-0.107$ \\
$\quad$ Length of care episode & 0.056 & 0.035 & 0.101 & -0.011 to 0.124 \\
Comorbidity & 0.840 & 0.223 & $<0.001^{*}$ & $0.400-1.278$ \\
IDU index change &
\end{tabular}

$C I$ confidence interval, $I D U$ inappropriate drug use, $S D$ standard deviation

$* p<0.05$ changes when care episodes are shorter or (2) the care episodes are shorter because patients need fewer drug changes. However, because (1) the length of care episodes decreased and (2) the quality of drug prescribing (according to the IDU index score) also decreased from 2005 to 2015 , it is unlikely that the length of the care episode was determined by the need to change prescriptions.

The present study indicates that scientific updates sometimes translate slowly into clinical practice. Although varying protocols to decrease inappropriate drug prescriptions have been developed over the years, the need for healthcare services to decrease the length of the care episode appears to have been prioritized, seemingly at the expense of the quality of care.

Given that the number of prescription changes is considered a quality factor in geriatric care, this major finding raises questions about how observed reductions in continuous care episode (in a geriatric clinic over the last decade) have affected the quality of patient care. Care episode reductions raise productivity but may result in loss of quality.

\subsection{Limitations}

Drugs related to the primary diagnosis of pneumonia were not included in the analysis as they were used only temporarily. Drugs administered as needed were likewise not included because they were not consistently registered throughout the study periods. This may have decreased the number of potential drug interactions.

As a model for a care episode for an average geriatric patient, we selected patients who were admitted for pneumonia treatment, but this is not an established method. That said, the criterion was applied because pneumonia is common, affects many old patients, and is a condition that does not promote changes in long-term prescriptions.

The study population was slightly older and had a higher percentage of males than the entire clinic population. This may be due to a higher percentage of study patients with COPD in the study, which is a risk factor for pneumonia. This suggests that study patients had more comorbidities than the average patient in the clinic. But because comorbidity, age, and sex were not found to be correlated with the endpoint prescription changes in this study, these differences may not be of great relevance.

Clinical severity is a factor that could influence prescription changes. However, it is difficult to extract clear and measurable assessments on clinical severity from medical journals, and we did not have access to that information. We used the CCI to assess comorbidities, although we are aware it does not reflect clinical severity.

Other changes not investigated in this study may have occurred during the study period and may have influenced prescription changes. For example, most study patients were transferred from other hospitals; consequently, they probably had shorter care episodes and may have been in a more severe or less stable condition when they arrived at 
the geriatric clinic in 2015 compared with patients in 2005 . The content of care at the geriatric clinic may also have changed in ways that we did not evaluate.

\section{Conclusion}

Study patients had significantly more comorbidities and prescriptions but were treated for a shorter time period and had fewer prescription changes in 2015 than in 2005. More prescription changes were significantly associated with longer care episodes and improved drug-prescribing quality as per the IDU index score. The association between geriatric care episodes and drug prescribing quality was positive in 2010 and negative in 2015 , according to the IDU index score.

Given that number of prescription changes is considered a quality factor within geriatric care, quality of care may have decreased along with the length of care episodes during the 2005-2015 study period.

\section{Compliance with Ethical Standards}

Conflicts of interest Marianne Reimers, Maria Eriksdotter, Åke Seiger, and Johan Fastbom have no conflicts of interest relevant to the content of this study.

Ethical Approval Ethical approval was received from the Regional Ethical Review Board in Stockholm.

Funding The study was supported by the Stockholms Sjukhem Foundation.

Open Access This article is distributed under the terms of the Creative Commons Attribution-NonCommercial 4.0 International License (http://creativecommons.org/licenses/by-nc/4.0/), which permits any noncommercial use, distribution, and reproduction in any medium, provided you give appropriate credit to the original author(s) and the source, provide a link to the Creative Commons license, and indicate if changes were made.

\section{References}

1. Craftman AG, Johnell K, Fastbom J, et al. Time trends in 20 years of medication use in older adults: findings from three elderly cohorts in Stockholm, Sweden. Arch Gerontol Geriatr. 2016;63:28-35. https://doi.org/10.1016/j.archger.2015.11.010 (epub 2015 Nov 28).

2. Haider SI, Johnell K, Thorslund M, et al. Trends in polypharmacy and potential drug-drug interactions across educational groups in elderly patients in Sweden for the period 1992-2002. Int J Clin Pharmacol Ther. 2007;45(12):643-53.

3. Guthrie B, Makubate B, Hernandez-Santiago V, et al. The rising tide of polypharmacy and drug-drug interactions: population database analysis 1995-2010. BMC Med. 2015;13:74. https://doi. org/10.1186/s12916-015-0322-7.

4. Wastesson JW, Fastbom J, Johnell K. Expanding the proportion of life with polypharmacy in Sweden: 2006-2013. J Am Dir Assoc. 2016;17:956-8.

5. Singh S, Bajorek B. Pharmacotherapy in the ageing patient: the impact of age per se. Ageing Res Rev. 2015;24:99-110. https:// doi.org/10.1016/j.arr.2015.07.006.

6. Lucas C, Byles J, Martin JH. Medicines optimisation in older people: taking age and sex into Account. Maturitas. 2016;93:114-20.

7. Astrand E, Astrand B, Antonov K, et al. Potential drug interactions during a three-decade study period: a cross-sectional study of a prescription register. Eur $\mathrm{J}$ Clin Pharmacol. 2007;63(9):851-9.

8. Teka F, Teklay G, Ayalew E, et al. Potential drug-drug interactions among elderly patients admitted to medical ward of Ayder Referral Hospital, Northern Ethiopia: a cross sectional study. BMC Res Notes. 2016;9:431. https://doi.org/10.1186/ s13104-016-2238-5.

9. Mallet L, Spinewine A, Huang A. The challenge of managing drug interactions in elderly People. Lancet. 2007;370:185-91.

10. Spinewine A, Schmader KE, Barber N, et al. Appropriate prescribing in elderly people: how well can it be measured and optimised? Lancet. 2007;370:173-84.

11. Klarin I, Wimo A, Fastbom J. The association of inappropriate drug use with hospitalisation and mortality: a populations-based study of the very old. Drugs Aging. 2005;22(1):69-82.

12. Schmader KE, Hanlon JT, Pieper CF, et al. Effects of geriatric evaluation and management on adverse drug reactions and suboptimal prescribing in the frail elderly. Am $\mathrm{J}$ Med. 2004;116-6:394-401.

13. Wickop B, Baeh M, Harterich S, et al. Potentially inappropriate medication use in multimorbid elderly inpatients: differences between the FORTA, PRISCUS and STOPP ratings. Drugs Real World Outcomes. 2016;3:317-25. https://doi.org/10.1007/ s40801-016-0085-2.

14. Kersten H, Hvidsten LT, Gløersend G, et al. Clinical impact of potentially inappropriate medications during hospitalization of acutely ill older patients with multimorbidity. Scand J Prim Health Care. 2015;33-4:243-51.

15. Dauphinot V, Faure R, Bourguignon L, et al. Factors associated with changes in exposure to anticholinergic and sedative medications in elderly hospitalized patients: multicenter longitudinal study. Eur J Neurol. 2017;24(3):483-90. https://doi.org/10.1111/ ene.13228.

16. Larsen MD, Rosholm JU, Hallas J. The influence of comprehensive geriatric assessment on drug therapy in elderly patients. Eur J Clin Pharmacol. 2014;70(2):233-9. https://doi.org/10.1007/ s00228-013-1601-x.

17. Charlson ME, Pompei P, Ales KL, et al. A new method of classifying comorbidity development in longitudinal prognostic studies and validation. J Chron Dis. 1987;40-5:373-83.

18. Indicators for good drug therapy in the elderly. Swedish National Board of Health and Welfare, 2017;23-39. http://www. socialstyrelsen.se/publikationer2017/2017-6-7.

19 Fastbom J, Johnell K. National indicators for quality of drug therapy in older persons: The Swedish experience from the first 10 years. Drugs Aging. 2015;32(3):189-99. 